\title{
Tuberculosis and COVID-19 Screening at Health Facilities: A Cross-Sectional Survey of Health Care Workers in Nigeria during the COVID-19 Pandemic
}

\author{
Chika A. Okoro ${ }^{*}$, Eridiong O. Onyenweaku², Emecheta G. Okwudire3 ${ }^{3}$ Muriel K. Kalu4, \\ Oluremilekun C. Kusimo ${ }^{1}$, Victor Williams ${ }^{5}$ \\ ${ }^{1}$ Communicable and Non-Communicable Diseases Cluster, World Health Organization, Owerri, Nigeria \\ ${ }^{2}$ Human Nutrition \& Dietetics Unit, Department of Biochemistry, University of Calabar, Calabar, Nigeria \\ ${ }^{3}$ Department of Radiology, Federal Medical Centre, Owerri, Nigeria \\ ${ }^{4}$ Department of Radiology, Ibom Specialist Hospital, Uyo, Nigeria \\ ${ }^{5}$ School of Public Health, Faculty of Health Sciences, University of the Witwatersrand, Johannesburg, South Africa \\ Email: ^drokoro2009@yahoo.com,contactdy@yahoo.com, meetle1@gmail.com,drmurielinyang@gmail.com, \\ stremi2001@yahoo.com,vw1415@hotmail.com
}

How to cite this paper: Okoro, C.A., Onyenweaku, E.O., Okwudire, E.G., Kalu, M.K., Kusimo, O.C. and Williams, V. (2021) Tuberculosis and COVID-19 Screening at Health Facilities: A Cross-Sectional Survey of Health Care Workers in Nigeria during the COVID-19 Pandemic. Journal of Tuberculosis Research, 9, 18-30. https://doi.org/10.4236/jtr.2021.91002

Received: February 1, 2021

Accepted: March 27, 2021

Published: March 30, 2021

Copyright (อ 2021 by author(s) and Scientific Research Publishing Inc. This work is licensed under the Creative Commons Attribution International License (CC BY 4.0).

http://creativecommons.org/licenses/by/4.0/ (c) (i) Open Access

\begin{abstract}
Background: The coronavirus disease 2019 (COVID-19) incidence continues to rise in many parts of the world with increasing fatality. At the same time, tuberculosis (TB) has been identified as the leading cause of death amongst all infectious diseases globally. Routine screening of clients visiting health facilities can help to prevent the spread of these diseases. Aim: To assess the relationship between the practice of facility-based routine tuberculosis screening and routine screening for COVID-19. Methodology: Using a Snowball technique, a cross-sectional online survey was carried out during the national lockdown from 5 July to 5 August 2020. The target population for this survey was health care workers from the different health facilities across Nigeria. An online semi-structured questionnaire was used to interview healthcare workers to identify their knowledge, attitudes, and practices (KAP) towards COVID-19 and the practice of routine TB screening. Descriptive analysis, analysis of variance (ANOVA), and Pearson's Chi-square test was used for statistical comparative analysis. Results: This shows that $53.9 \%$ of healthcare workers did not practice routine TB screening while $46.9 \%$ did not practice routine COVID-19 screening. Respondents who practiced routine TB screening were found to be more likely to practice routine COVID-19 screening $(\mathrm{p}<0.001)$. Healthcare workers in primary healthcare centers were more likely to carry
\end{abstract}


out routine screening for both diseases $(\mathrm{p}<0.001)$ and among these, Community Healthcare Workers were more likely to carry out routine screening for both diseases than other cadres $(\mathrm{p}<0.001)$. Conclusion/Recommendation: Routine screening for infectious diseases is still not institutionalized in the Nigerian health system, making the control of these diseases difficult. Continued sensitization on the need for routine screening for infectious diseases like TB and COVID-19 should be done for healthcare workers at the different levels in the health care system.

\section{Keywords}

COVID-19, Tuberculosis, Screening, Preventive Measures, Healthcare

\section{Introduction}

World Health Organization (WHO) reported the outbreak of the novel coronavirus disease for the first time on the 31st December 2019 and went on to declare it a pandemic on the $11^{\text {th }}$ of March, 2020 [1]. The Severe Acute Respiratory Syndrome coronavirus-2 (SARS-CoV-2) has been demonstrated to cause mild to severe respiratory disease that could be fatal in the elderly and persons with co-morbidities [2]. As of October $12^{\text {th }}, 2020$, the weekly updates by WHO showed 37,109,851 persons had been infected globally with 1,070,355 deaths and an estimated case fatality rate of $2.9 \%$ globally [1]. The total number of cases reported in Nigeria in the same period was 60,430 with 1115 deaths recorded giving a case fatality rate of $1.8 \%$ for the country [3]. The symptoms of the disease include fever, respiratory distress, fatigue, dry cough, and in some cases, loss of smell and taste [1]. A high number of people who get infected with COVID-19 remain asymptomatic [1]. These asymptomatic persons are more likely to be the source of transmission of the disease in the community if they do not follow protocols for the prevention of COVID-19 [1] which has been characterized by high morbidity and mortality rates [4].

Similarly, tuberculosis is also an infectious disease which also primarily causes respiratory distress and is caused by mycobacterial species [5]. About one-third of the world population is infected with latent tuberculosis globally and can be on this latent phase all their lives without developing active TB disease except they become immunocompromised [5]. However, a significant percentage of the infected persons go on to develop active TB disease and have the potential to spread the disease from one person to another [6]. People with HIV or other immunosuppressive conditions are more likely to develop active TB from a latent state compared to those who are immunocompetent [6]. The natural history of TB is such that almost all who develop TB disease will eventually die of the disease if appropriate treatment is not instituted [7]. This contrasts with the natural history of COVID-19 where the majority of the persons who develop the disease recover spontaneously [8]. 
Tuberculosis has been identified as the leading infectious disease killer in the world [9]. According to the 2019 Global TB report, an estimated 10 million people fell ill with TB in 2018 with 7 million notified, leaving a gap of about 3 million persons with TB disease who are yet to be identified and placed on treatment. About 1.451 million persons, 251,000 of which were people living with HIV, were said to have died in the same year [9]. Nigeria is among the 10 high burden countries that contributed $80 \%$ of the 3 million gap in case notification accounting for $8 \%$ of the unidentified cases with an estimated 300,000 unidentified TB cases in the country [9].

Tuberculosis continues to have a devastating impact on the health systems and economies of several countries including Nigeria. The Nigerian health system was already overburdened before the COVID-19 outbreak with deficient infrastructure and human resource capacity to deal with already existing diseases [10]. This simply implies that basic preventive measures like routine screening for common infectious diseases could play a significant role in the control of both COVID-19 and TB. Screening for both diseases requires the use of a standardized symptoms checklist as recommended by the Federal Ministry of Health.

Nigeria has been making efforts to institutionalize routine TB screening in all health facilities in the country. In 2018, the National Council of Health made it mandatory that all health facilities in the country must begin to routinely screen all their clients for TB as a key strategy for eliminating the disease by 2035 as targeted in sustainable development goal 3 [11]. Official memos were sent out to Commissioners of Health in the 36 states of the Federation by the Minister of Health mandating them to hold heads of facilities responsible for implementing this policy [12].

To screen a client for tuberculosis, a healthcare worker is expected to ask a client questions relating to a standardized symptoms checklist that seeks to know whether the client has had a cough of two weeks or more, weight loss, drenching night sweats, loss of appetite and persistent low-grade fever [13] [14]. Any client coughing for 2 weeks or more with or without any of the other symptoms is considered a "presumptive TB case" and is referred for sputum analysis or X-ray [13]. To screen for COVID-19, a health worker is expected to ask questions related to the occurrence of the following symptoms: fever, respiratory distress, fatigue, dry cough, loss of smell, and taste [1]. Symptomatic clients are then referred for testing. Given the above, this study seeks to evaluate the extent of routine facility-based TB screening practiced by Nigerian health workers and whether the practice has any relationship with the practice of routine COVID-19 screening in the facilities following the outbreak of the pandemic.

\section{Methodology}

The study employed quantitative methodology and data was collected by means 
of an online-cross-sectional survey.

\subsection{Study Population}

The population for the cross-sectional study consisted of 426 respondents (all healthcare workers) from 29 states in Nigeria.

\subsection{Sample Size}

Using a confidence interval of 5\% and a confidence level of $95 \%$, the sample size for the survey was calculated using the formula:

$$
N=z^{2} \times p \times(1-p) / c^{2}
$$

where $N=$ sample size, $Z=$ value corresponding to a given confidence level (1.96 at $95 \%$ level), $p=$ percentage of primary indicator taken as 0.5 and $c=$ standard error taken as 0.05 at $5 \%$ confidence interval.

The calculated sample size was 384 with a $10 \%$ adjustment for non-response getting it to 422 . A total of 426 responses from 29 states were analyzed for the survey.

\subsection{Sampling Procedure}

This study is a cross-sectional survey conducted in Nigeria using a snowball sampling approach. The online questionnaire link was sent to prospective respondents by the investigators via emails, WhatsApp, and other social media handles. Prospective respondents were encouraged to share the survey with their contacts and other online platforms. Thus, the link was forwarded to other people apart from the first respondents.

The online survey was conducted during the latter phase of the COVID-19 pandemic (between June and August 2020) when the lockdown was eased by the Nigerian government to allow interstate movement and some restricted gatherings with strict observance of COVID-19 protocols. The online survey was selected for this study since a population-based survey was not feasible under the current social restrictions due to COVID-19.

The study population included only individuals with access to the internet. Respondents who understood the English language and were 18 years old and above, were recruited for the study. Respondents were healthcare workers such as doctors, nurses, pharmacists, laboratory scientists, community health workers, and records staff pooled from 29 states in Nigeria. Being an online study using a Snowball sampling method, the survey allowed respondents from many states in Nigeria to participate. Participation in this survey was anonymous, consensual, and voluntary.

\subsubsection{Questionnaire Design and Administration}

A semi-structured questionnaire was designed and converted to the online version using Google forms. The survey instrument used in this study was designed according to the guidelines recommended for the awareness and prevention of 
COVID-19 by the Nigeria Centre for Disease Control [3], and from the KAP of previous infectious disease outbreaks (Ebola and Lassa fever) in Nigeria [15] [16]. The online questionnaire was reviewed by Public health and epidemiology experts on the relevance, correctness, and relativity of the instrument to COVID-19 and tuberculosis, particularly considering the study population. The finalized questionnaire was pretested on 10 participants and these were excluded from the study.

The finalized questionnaire contained questions assessing socio-demographics, knowledge, attitudes, perceptions, and practices of the respondents regarding the coronavirus pandemic and screening for TB. A sample of this questionnaire is attached as supplementary material.

\subsubsection{Informed Consent and Data Privacy}

Respondents were asked to carefully read and understand the content summary before proceeding to the questionnaire. Informed consent was obtained for all participants. Participants' responses were anonymous and confidential according to Google's privacy policy (https://policies.google.com/privacy?hl=en). Participants were not permitted to provide their names or contact information. Additionally, participants were able to end study participation and leave the questionnaire at any stage before the submission process; if doing so, their responses would not be saved. Responses were saved only by clicking on the provided "submit" button. By completing the survey, participants acknowledged their voluntary consent to participate in this anonymous study.

\subsubsection{Ethical Clearance}

Ethical clearance was obtained for the survey from the Research Ethics Committee of the University of Calabar, Calabar, Nigeria.

\subsection{Data Analysis}

Descriptive statistics such as frequencies, percentages, and charts, were used to define the proportion of responses for each question and the total distribution in the total score of each questionnaire. All statistical analyses were performed using the Statistical Package for Social Sciences software (SPSS, version 20.0) and Microsoft Excel 2010. Values were computed and reported as mean \pm SD (standard deviation). The level of statistical significance was set at $\mathrm{p}$ values below 0.05. Bivariate analysis using the Chi-square test was applied to find the relationship between routine TB and COVID-19 screening, occupation, facility level, and facility type.

\section{Results}

\subsection{Socio-Demographics Characteristics of the Surveyed Population}

Table 1 presents the socio-demographic characteristics of the surveyed participants (426 people). There were more males (56.3\%) mostly aged 36 and 49 years 
Table 1. Socio-demographic characteristics of the surveyed population $(\mathrm{N}=426)$.

\begin{tabular}{|c|c|c|c|}
\hline Variable & Sub-variable & Frequency & Percentage \\
\hline \multirow{3}{*}{ Sex } & Male & 240 & 56.3 \\
\hline & Female & 186 & 43.7 \\
\hline & Total & 426 & 100 \\
\hline \multirow{5}{*}{ Age group } & $18-25$ years & 5 & 1.2 \\
\hline & $36-45$ years & 112 & 26.3 \\
\hline & $36-49$ years & 245 & 57.5 \\
\hline & 50 years \& above & 64 & 15.0 \\
\hline & Total & 426 & 100 \\
\hline \multirow{8}{*}{ Occupation } & Doctor & 251 & 58.9 \\
\hline & Nurse & 44 & 10.3 \\
\hline & Community Health Worker & 62 & 14.6 \\
\hline & Laboratory scientist & 26 & 6.1 \\
\hline & Pharmacist & 16 & 3.8 \\
\hline & Dietitian & 6 & 1.4 \\
\hline & Others & 21 & 5.0 \\
\hline & Total & 426 & 100 \\
\hline \multirow{4}{*}{ Position } & Management & 6 & 1.4 \\
\hline & Service delivery & 406 & 95.3 \\
\hline & Both & 14 & 3.3 \\
\hline & Total & 426 & 100 \\
\hline \multirow{4}{*}{ Facility Level } & Primary (Health centres) & 107 & 25.1 \\
\hline & Secondary (Hospitals/clinics) & 100 & 23.5 \\
\hline & $\begin{array}{l}\text { Tertiary (Teaching/Specialist } \\
\text { hospitals, Federal Med. centres) }\end{array}$ & 219 & 51.4 \\
\hline & Total & 426 & 100 \\
\hline \multirow{5}{*}{ Facility Type } & Faith-based & 12 & 2.8 \\
\hline & Non-Governmental Org. (NGO) & 24 & 5.6 \\
\hline & Private & 74 & 17.4 \\
\hline & Public & 316 & 74.2 \\
\hline & Total & 426 & 100 \\
\hline
\end{tabular}

(57.5\%). More than half of the respondents were doctors (58.9\%), followed by community health workers (14.6\%), nurses (10.3\%), and pharmacists (3.8\%). Others who participated in the study were medical laboratory scientists and dietitians. Almost all the respondents (95.3\%) reported being involved in service delivery. Due to the geographical locations of the authors, the Southern region of the country recorded more responses namely: Imo (31.9\%-South East), Cross River (16.4\%-South-South), Akwa Ibom (11.5\%-South-South), and Lagos (4.5\%-South West), but a good number of responses (35.7\%) also came from all the other regions (see Table 2). The study widely covered healthcare workers 
Table 2. Responses by region and state.

\begin{tabular}{ccc}
\hline Variable Description & Number of Responses & Percentage (\%) \\
\hline Region & 6 & 1.4 \\
North-East & 39 & 9.2 \\
North-Central & 46 & 10.8 \\
North-West & 161 & 37.8 \\
South-East & 136 & 31.9 \\
South-South & 38 & 8.9 \\
South-West & & \\
State & 49 & 11.5 \\
Akwa Ibom & 70 & 16.4 \\
Cross River & 26 & 6.1 \\
Federal Capital Territory & 136 & 31.9 \\
Imo & 25 & 5.9 \\
Kano & 19 & 4.5 \\
Lagos & 101 & 32.5 \\
Others & &
\end{tabular}

located and working in various parts of Nigeria.

\subsection{Knowledge of COVID-19}

The studied population appeared to have a good knowledge of COVID-19 and its mode of transmission (see Figure 1). From the results, most of the respondents $(97.7 \%)$ correctly identified COVID-19 as being caused by a virus with about $96.2 \%$ of respondents correctly stating that COVID-19 was transmitted through contact with infected droplets. About a third (33.8\%) of respondents thought it to be airborne, 5.9\% thought it was transmitted through contaminated food, while $4.3 \%$ stated it was sexually transmitted albeit, wrongly. Doctors were more likely to choose air as the mode of transmission and this was found to be statistically significant $(\mathrm{p}=0.008)$. No significant relationship was found between occupation and choosing droplets, sexual intercourse, faeco-oral, contaminated foods, close contact, contaminated surfaces, or dirty hands as modes of transmission of COVID-19 ( $\mathrm{p}>0.05)$.

Greater than four-fifths (86\%) of all respondents identified handwashing, use of sanitisers, facemasks, social distancing, and restriction of mass gatherings as effective measures for preventing the spread of COVID-19. A good proportion of the respondents identified cough $(40.6 \%)$, loss of appetite $(21.7 \%)$, respiratory distress $(15.1 \%)$, and fever $(8.1 \%)$ as the commonest symptoms of COVID-19. Also, the majority of the respondents (84.2\%) identified nasopharyngeal swab as the sample used in testing for COVID-19, while $8.8 \%$ and $7.1 \%$ wrongly identified blood and sputum samples respectively, as the test sample. Most of the respondents $(89.7 \%)$ admitted to knowing where to refer patients with symptoms suggestive of COVID-19 with $2.8 \%$ stating they did not know. No significant 


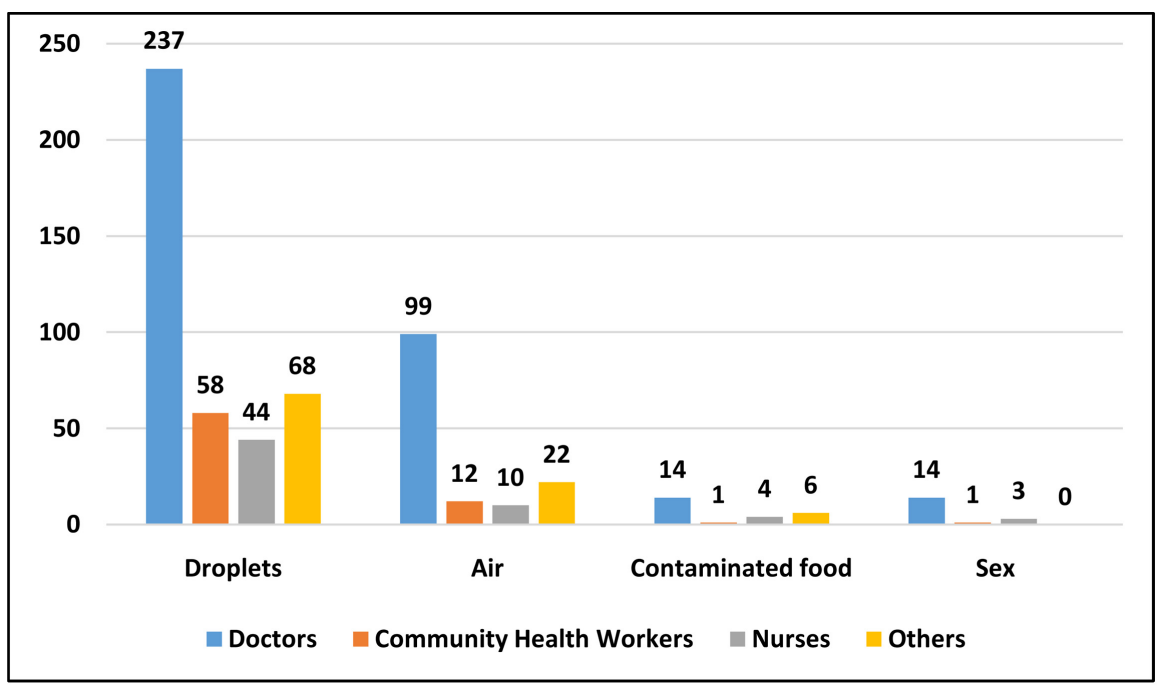

Figure 1. Knowledge of respondents on the possible mode of transmission of COVID-19.

relationship was found between occupation, type of facility or facility level, and knowledge of where to refer patients for COVID-19 testing ( $p>0.05$ ). Respondents' detailed knowledge of Tuberculosis was not assessed during the survey.

\subsection{Screening for Tuberculosis and COVID-19}

More than half (54.7\%) of respondents stated that routine TB screening was not done for clients in their facilities while $46.9 \%$ similarly stated routine screening of clients for COVID-19 was not done. About $26.5 \%$ and $35.7 \%$ of respondents stated that they carried out the routine screening of clients for TB and COVID-19 in their facilities, respectively. Very few (3.8\%) of the respondents stated they did not know if routine TB screening was carried out in their facility (see Figure 1 \& Figure 2). Among the respondents who agreed they conducted routine $\mathrm{TB}$ screening in their facilities, $61.1 \%$ of them stated they conduct COVID-19 screening at the same time, while $45.4 \%$ of those who stated they conducted routine screening for COVID-19 agreed they also conducted routine TB screening for clients at the same time (see Figure 3). This was found to be statistically significant $(\mathrm{p}<0.001)$.

A strong correlation was found between occupation, facility level and type of facility, and the practice of routine screening for both TB and COVID-19. Community health extension workers $(71.1 \%, 73.6 \%)$, health workers at primary levels $(60.7 \%, 60.7 \%)$, and healthcare workers (HCWs) in public facilities $(27.5 \%, 35.8 \%)$ were more likely to carry out routine screening for TB and COVID-19 in their facilities respectively ( $\mathrm{p}<0.001$ for all three). Only $11.4 \%$ of respondents from tertiary facilities conducted routine $\mathrm{TB}$ and screening in their facilities compared to $27.9 \%$ for COVID-19. Doctors and nurses were more likely to carry out routine COVID-19 screening in their facilities than routine TB screening. Less than a third (29.1\%) of doctors and $44.2 \%$ of nurses stated they carried out routine COVID-19 screening in their facilities while $13.1 \%$ and 


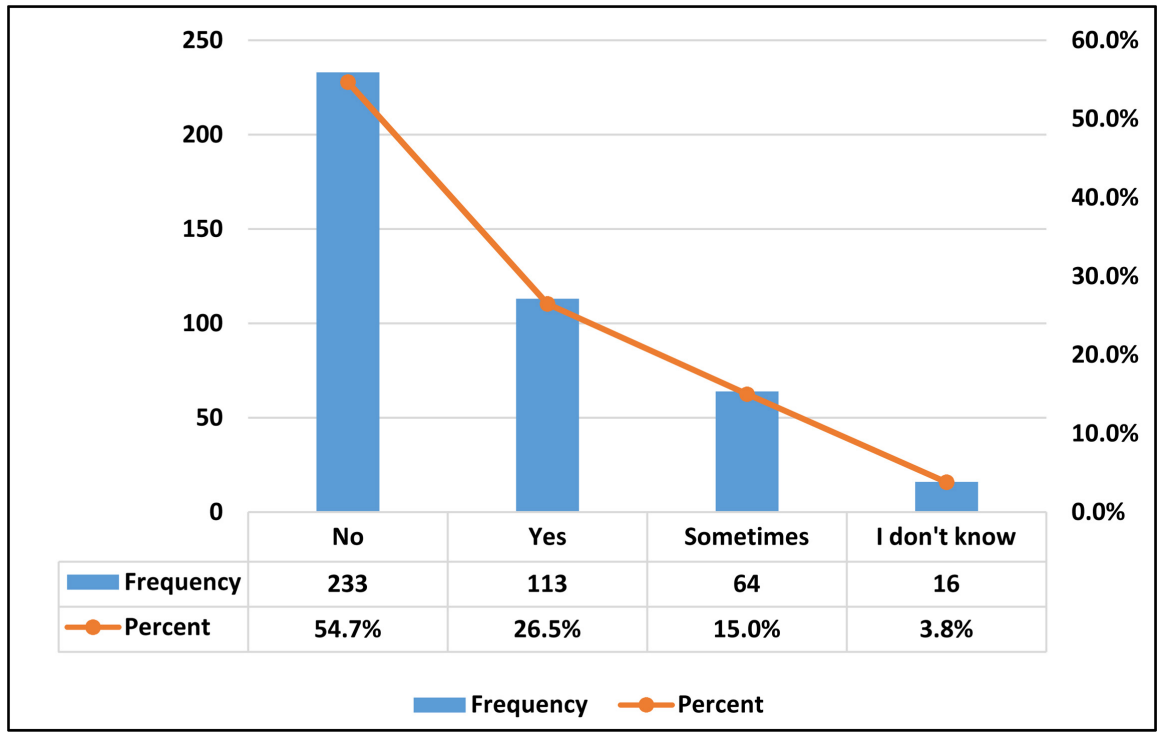

Figure 2. Frequency of routine TB screening among all respondents.

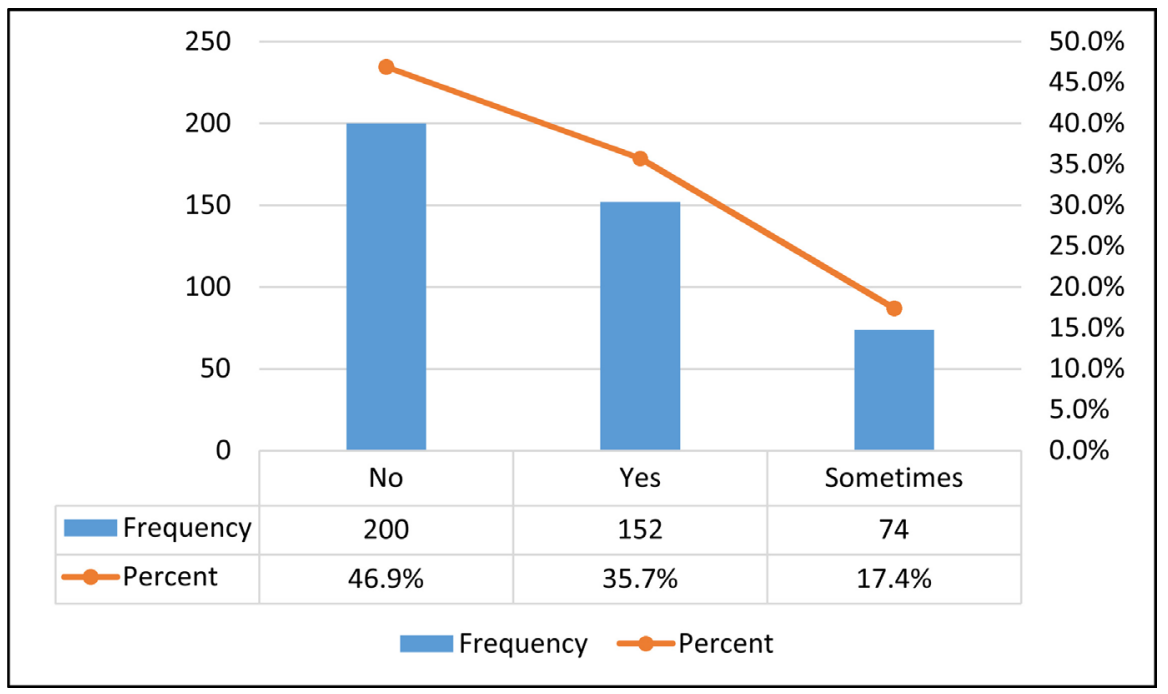

Figure 3. Frequency of routine COVID-19 screening among all respondents.

$27.5 \%$ respectively admitted to carrying out routine $\mathrm{TB}$ screening in their facilities $(\mathrm{p}<0.001)$.

\section{Discussion}

The respondents showed a good knowledge of COVID-19 and a high level of use of preventive measures against it. On the contrary, a poor attitude towards the disease and to work was adopted by most of the respondents during the pandemic. Studies in Pakistan [17] and Vietnam [18] reported similar findings of a high level of knowledge among HCW. This agrees with our findings in Nigeria. This could be due to the wide publicity on COVID-19 globally with tailored information for different population groups' especially healthcare workers. A study by Bhagavathula et al. [19], however, reported a low level of knowledge of 
COVID-19 among HCWs in various geographic regions worldwide. This may most likely be due to the fact the study was conducted in early March 2020 when there was little awareness of COVID-19 and most countries were yet to commence a lockdown.

The difference in practice between staff at Primary Health Care (PHC) level and those at the secondary and tertiary levels could be explained by the fact that most infectious disease programs are targeted at health workers at that level leaving those at the higher levels with minimal information on the possible assumption that they ought to know. The majority of sensitization and training on the control of infectious diseases like Tuberculosis usually involves people at the PHC level. This raises their awareness levels and indices of suspicion. The higher likelihood of doctors and nurses doing more routine screening for COVID-19 than tuberculosis may be related to the level of information available to them following the intense awareness creation around the pandemic, and the acuteness of COVID-19 compared to TB which is a chronic disease and a "Silent Killer". The fear of contracting COVID-19 from clients could also be a motivation for routine screening.

The observed low level of routine screening for tuberculosis among health workers generally may be related to a lack of knowledge of the effectiveness of early diagnosis and treatment in limiting the spread of the disease [20]. It may also be because of the attitude of the health workers who believe TB screening should be left to the designated officers at the TB treatment units in their facilities. The inadequate screening for COVID-19 could be attributed to the unavailability of proximal testing equipment and centres as is currently seen in the country. For these two diseases, the fear of being stigmatized, even at the facility level, may prompt clients to decline providing answers to questions related to the diseases thereby making screening difficult for health workers [21].

Most of the respondents who regularly conducted routine TB screening before the outbreak of the pandemic also showed a higher tendency to routinely screen for COVID-19 as reflected in the results. This will most likely be attributed to the fact that the healthcare workers were already primed to routinely screen for the presence of signs and symptoms of common infectious diseases.

\section{Limitation}

This study was limited to an online survey because of the COVID-19 lockdowns which made it impossible for the researchers to move to certain rural/semi-urban settings to include participants from such areas. As a result, the study reflects responses from a socio-economic class of health care workers who can access internet facilities and had smartphones/laptops. Also, the validity of answers, which is a general problem of online surveys, may be difficult to ascertain. In our analysis, we did not conduct advanced statistical analysis such as a multilevel analysis to ascertain if working at a certain level of a health facility or state had any effect on our findings. But this was compensated for by the different com- 
parative analyses which gave good estimates for the different groups of healthcare workers and health facilities.

\section{Conclusion}

From our survey, there is a good knowledge of COVID-19 and its preventive measures among the surveyed health care workers. We noted that routine screening for TB and COVID-19 is not being practised in most health facilities in Nigeria and that the practice of routine screening for one infectious disease could lead to a health worker routinely screening for other infectious diseases as seen in the high percentage of healthcare workers who screened for both TB and COVID-19. Despite being made a policy and pronounced as the standard of care, routine TB screening is yet to be institutionalized in the Nigerian health system after nearly 3 years. There is the need to fully engage all cadres of healthcare workers to inform and educate them on the relevance of routine screening of clients in the control of infectious diseases. Adequate infrastructure and diagnostic equipment, including well-designed hospital environments, should be made available as part of measures to encourage routine screening of clients for infectious diseases, especially tuberculosis.

\section{Authors' Contributions}

Dr. C. Okoro designed the study, produced the Microsoft word version of the questionnaire, participated in the extraction and collation of the google responses, and coordinated the research and statistical analyses. Dr. E. Onyenweaku co-designed the study, created the online questionnaire, participated in the extraction and collation of the google responses, and wrote the first draft of the manuscript. Dr. E. Okwudire participated in data extraction and collation of google responses, data analysis and made contributions to the research article. Dr. M. Kalu participated in data extraction and made contributions to the research article. Dr. O. Kusimo and Dr. V. Williams made contributions to the research article, gave inputs on the general structure and content. All the authors read and approved the final manuscript.

\section{Conflicts of Interest}

The authors declare no competing interests exist.

\section{References}

[1] World Health Organization (WHO) (2020) Coronavirus Disease (COVID-19) Situation Report-126.

https://www.who.int/docs/default-source/coronaviruse/situation-reports/20200525covid-19-sitrep-126.pdf?sfvrsn=887dbd66_2

[2] Abdelhafiz, A.S., Mohammed, Z., Ibrahim, M.E., Ziady, H.H., Alorab, M., Ayyad, M., et al. (2020) Knowledge, Perceptions, and Attitude of Egyptians towards the Novel Coronavirus Disease (COVID-19). Journal of Community Health, 45, 881-890. https://doi.org/10.1007/s10900-020-00827-7 
[3] Nigerian Centre for Disease Control (NCDC) (2020) Frequently Asked Questions on Coronavirus-29/02/20. https://covid19.ncdc.gov.ng/faq

[4] Roy, D., Tripathy, S., Kar, S.K., Sharma, N., Verma, S.K. and Kaushal, V. (2020) Study of Knowledge, Attitude, Anxiety and Perceived Mental Healthcare Need in Indian Population during COVID-19 Pandemic. Asian Journal of Psychology, 51, Article ID: 102083. https://doi.org/10.1016/j.ajp.2020.102083

[5] Adam, C., Victor, D.M., Thomas, S. and Christian, W. (2019) The Global Prevalence of Latent Tuberculosis: A systematic Review and Meta-Analysis. European Respiratory Journal, 54, Article ID: 1900655. https://doi.org/10.1183/13993003.00655-2019

[6] Bell, L.C.K. and Noursadeghi, M. (2018) Pathogenesis of HIV-1 and Mycobacterium tuberculosis Co-Infection. Nature Reviews Microbiology, 16, 80-90.

https://doi.org/10.1038/nrmicro.2017.128

[7] Lawn, S.D., Wood, R. and Wilkinson, R.J. (2011) Changing Concepts of "Latent Tuberculosis Infection" in Patients Living with HIV Infection. Clinical and Developmental Immunology, 2011, Article ID: 980594.

https://doi.org/10.1155/2011/980594

[8] Li, J.Y., You, Z., Wang, Q., et al. (2020) The Epidemic of 2019-Novel-Coronavirus (2019-nCoV) Pneumonia and Insights for Emerging Infectious Diseases in the Future. Microbes and Infection, 22, 80-85.

https://doi.org/10.1016/j.micinf.2020.02.002

[9] World Health Organization (WHO) (2020) Global TB Report 2019.

https://www.who.int/teams/global-tuberculosis-programme/tb-reports/global-repor $\underline{\mathrm{t}-2019}$

[10] Adeloye, D., David, R., Olaogun, A.A., et al. (2017) Health Workforce and Governance: The Crisis in Nigeria. Human Resources for Health, 15, 32. https://doi.org/10.1186/s12960-017-0205-4

[11] United Nations (2015) Sustainable Development Goals. https://www.un.org/development/desa/disabilities/envision2030.html

[12] Federal Ministry of Health (2018) Implementation of 60th National Council of Health Resolution on Compulsory TB Notification by Private Facilities in Nigeria.

[13] Owiti, P., Onyango, D., Momanyi, R. and Harries, A.D. (2019) Screening and Testing for Tuberculosis among the HIV-Infected: Outcomes from a Large HIV Programme in Western Kenya. BMC Public Health, 19, 29.

https://doi.org/10.1186/s12889-018-6334-4

[14] Ejaz, Q., Razia, F., Mahboob, U.H., et al. (2017) Yield of Facility-Based Verbal Screening amongst Household Contacts of Patients with Multi-Drug Resistant Tuberculosis in Pakistan. Journal of Clinical Tuberculosis and Other Mycobacterial Diseases, 7, 22-27.

[15] Etokidem, A.J., Ago, B.U., Mary, M., Affiong, E., Eno, U. and Anastasia, I. (2018) Ebola Virus Disease: Assessment of Knowledge, Attitude and Practice of Nursing Students of a Nigerian University. African Journal of Health Science, 18, 55-65. https://doi.org/10.4314/ahs.v18i1.9

[16] Reuben, C.R. and Gyar, S.D. (2016) Knowledge, Attitudes and Practices of Lassa Fever in and around Lafia, Central Nigeria. International Journal of Public Health and Epidemiology Research, 2, 14-21.

[17] Saqlain, M., Munir, M.M., Ur Rehman, S., et al. (2020) Knowledge, Attitude, Practice and Perceived Barriers among Healthcare Professionals Regarding COVID-19: A Cross-Sectional Survey from Pakistan. Journal of Hospital Infection, 105, 
419-423. https://doi.org/10.1016/j.jhin.2020.05.007

[18] Giao, H., Le An, P., Thi Ngoc han, N., Van Khanh, T., et al. (2020) Knowledge and Attitude towards COVID-19 among Healthcare Workers at District 2 Hospital, HO Chi Minh City. Asian Pacific Journal of Tropical Medicine, 13, 6-11. https://doi.org/10.4103/1995-7645.280396

[19] Bhagavathula, A.S., Aldhaleei, W.A., Rahmani, J., et al. (2020) Knowledge and Perceptions of COVID-19 among Healthcare Workers: Cross-Sectional Study. JMIR Public Health and Surveillance, 6, e19169. https://doi.org/10.2196/preprints.19160

[20] Wang, J., Fei, Y., Shen, H. and Xu, B. (2008) Gender Difference in Knowledge of Tuberculosis and Associated Healthcare Seeking Behaviors: A Cross-Sectional Study in a Rural Area of China. BMC Public Health, 8, 354.

https://doi.org/10.1186/1471-2458-8-354

[21] Oladimeji, O., Tsoka-Gwegweni, J.M., Adeyinka, D.A., Makola, L., Mitonga, K.H., Udoh, E.E., et al. (2018) Knowledge, Attitude and Perception of Tuberculosis Management among Tuberculosis-Infected Patients in Resource Constraint Setting: Field Experience from Oyo State, South-West, Nigeria. International Journal of Community Medicine and Public Health, 5, 1694-706.

https://doi.org/10.18203/2394-6040.ijcmph20181379

\section{Link to Survey Questionnaire}

https://docs.google.com/forms/d/1NKknoh97LwjY1Ks3y8kPkwyyq9mKI5gZ2N $\underline{\text { OJSYH8_1o/edit? usp=sharing_eil\&invite=CJrBtJAL\&ts=5ef1de0f. }}$ 